\title{
Retinal Vessel Density Changes on Optical Coherence Tomography Angiography and Predictive Factors in Normal-Tension Glaucoma Treated with Topical Beta-Blocker
}

\author{
Yun-Hsuan Lin ${ }^{1,2,3,+}+\mathbb{D}$, Shih-Ming Huang ${ }^{3,4,+}$, Lan-Hsin Chuang ${ }^{2,3}$ and Lung-Chien Chen ${ }^{1, *} \mathbb{D}$ \\ 1 Department of Electro-Optical Engineering, National Taipei University of Technology, Taipei 10608, Taiwan; \\ mabelle0527@gmail.com \\ 2 Department of Ophthalmology, Chang Gung Memorial Hospital, Keelung 20401, Taiwan; \\ lanhsin.chuang@gmail.com \\ 3 College of Medicine, Chang Gung University, Taoyuan 33302, Taiwan; skiwalkergg@gmail.com \\ 4 Department of Radiation Oncology, Chang Gung Memorial Hospital, Keelung 20401, Taiwan \\ * Correspondence: ocean@ntut.edu.tw; Tel.: +886-2-27712171 (ext. 4634); Fax: +886-2-87733216 \\ + Yun-Hsuan Lin and Shih-Ming Huang contributed equally.
}

Citation: Lin, Y.-H.; Huang, S.-M.; Chuang, L.-H.; Chen, L.-C. Retinal Vessel Density Changes on Optical Coherence Tomography Angiography and Predictive Factors in NormalTension Glaucoma Treated with Topical Beta-Blocker. Sci. Pharm. 2021, 89, 40. https://doi.org/10.3390/ scipharm 89030040

Academic Editors: Francisco Javier Otero-Espinar and Susi Burgalassi

Received: 21 July 2021

Accepted: 23 August 2021

Published: 25 August 2021

Publisher's Note: MDPI stays neutral with regard to jurisdictional claims in published maps and institutional affiliations.

Copyright: (C) 2021 by the authors Licensee MDPI, Basel, Switzerland. This article is an open access article distributed under the terms and conditions of the Creative Commons Attribution (CC BY) license (https:// creativecommons.org/licenses/by/ $4.0 /)$.

\begin{abstract}
Background: Topical antiglaucoma medications may alter the microcirculation in the optic nerve head. We aimed to evaluate the changes in retinal vessel density (VD) on optical coherence tomography angiography (OCTA) in patients with newly diagnosed normal-tension glaucoma (NTG) treated with a topical beta-blocker. (2) Methods: This study included 80 patients diagnosed with NTG not using systemic medication, who received topical carteolol treatment between December 2019 and November 2020. We studied the changes in the OCTA VD/signal strength index (SSI) after the 6-month treatment period and determined the predictive factors affecting the changes in VD/SSI. (3) Results: After the 6-month treatment period, the peripapillary VD increased in 40 patients but decreased in the other 40 patients. The univariate and multivariate analyses revealed that old age and hypertension were significant factors associated with a VD/SSI decrease after carteolol treatment. Moreover, high baseline peripapillary, superficial, and deep macular VDs were significantly associated with the VD decrease after carteolol treatment. (4) Conclusions: Carteolol treatment could increase or decrease the VD in patients with NTG. The baseline VD, age, and hypertension could affect these VD changes. Patients with NTG and higher baseline peripapillary or macular VD, older age, and hypertension are more likely to have a decreased VD after carteolol treatment.
\end{abstract}

Keywords: optical coherence tomography angiography; normal-tension glaucoma; vessel density; beta-blocker; carteolol; age; hypertension

\section{Introduction}

Normal-tension glaucoma (NTG) is a chronic, progressive optic neuropathy that leads to specific glaucomatous visual field (VF) defects [1]. Several studies have indicated that vascular effects play a crucial role in eyes with NTG [2]. Although many mechanisms underlying an abnormal ocular blood flow in NTG have been proposed, the exact mechanism remains unclear [3]. One of the most widely accepted hypotheses suggests that NTG results from damage due to low or fluctuating ocular blood flow and reperfusion injury in the optic nerve head $(\mathrm{ONH})[4]$.

Many tools are currently available for diagnosing and monitoring eyes with NTG. Among them, optical coherence tomography angiography (OCTA) enables a fast and noninvasive evaluation of the microvasculature and vessel density (VD) of various retinal layers. OCTA uses the laser light reflectance of the surface of moving red blood cells to accurately depict vessels through the retina. The diagnostic value of OCTA in eyes with high-tension glaucoma and NTG has been well demonstrated in recent years. In particular, 
many studies have reported a loss in VD during both the development and progression of glaucoma [5-7].

However, the effects of antiglaucoma medications on VD changes in eyes with glaucoma remain unclear. Topical $\beta$-adrenergic antagonists, when used in glaucoma treatment, function by inhibiting cyclic adenosine monophosphate production in the ciliary epithelium, with a corresponding decrease in aqueous humor secretion of $20-50 \%$ and a $20-30 \%$ IOP reduction. Carteolol is a $\beta$-adrenergic antagonist that has been widely used for many years in the treatment of simple ocular hypertension (OHT) and glaucoma [8,9]. Based on a previous report, peripapillary VD may be enhanced by the topical Rho-assisted coiled-coil forming protein kinase inhibitor ripasudil in eyes with primary open-angle glaucoma (POAG) and ocular hypertension [9]. An enhanced retinal VD may also be beneficial to visual function in glaucomatous eyes. In this study, we evaluated the changes in peripapillary VD on OCTA in patients with newly diagnosed NTG treated using topical carteolol.

\section{Materials and Methods}

This retrospective, single-center, observational study was conducted between December 2019 and November 2020 and included patients with newly diagnosed NTG who visited the glaucoma clinic of the Keelung branch of Chang Gung Memorial Hospital, Taiwan. The study was approved by the Institutional Review Board of Chang Gung Memorial Hospital (IRB approval number: 202000935B0). Written informed consent was obtained from all the patients. The study adhered to the principles of the Declaration of Helsinki.

\subsection{Patients}

Patients with newly diagnosed NTG who received 2\% topical carteolol hydrochloride (Mikelan LA Ophthalmic solution, Tokushima, Japan, once a day) were included in this study. The diagnosis of NTG was based on the following criteria of the European Glaucoma Society [1]: (1) patients older than 35 years, (2) normal intraocular pressure (IOP) less than $21 \mathrm{mmHg}$ without treatment, (3) ONH damage typical of glaucoma, (4) VF defects typical of glaucoma, (5) open anterior chamber angle on gonioscopy, and (6) no history of steroid use. All patients underwent detailed ophthalmologic examinations, including visual acuity assessment, Goldmann applanation tonometry, gonioscopy, slit-lamp biomicroscopy, central corneal thickness (CCT) measurement, standard automated perimetry (Swedish interactive threshold algorithm standard 30-2 test; Humphrey Field Analyzer II, Carl Zeiss Meditec, Dublin, CA, USA), and OCTA measurements (Optovue Inc., Fremont, CA, USA), before treatment and 6 months after topical carteolol treatment.

The exclusion criteria were as follows: patients taking antiglaucoma medication or any systemic medications before the study, patients with a history of intraocular surgery, patients with intraocular eye diseases other than NTG or cataracts, patients with unreliable VF test results (i.e., false-positive errors $>15 \%$ or fixation loss $>25 \%$ ), or OCTA signal strength index $(\mathrm{SSI})<40 \%$. In addition, patients who changed their topical medication or received laser treatment during the study period were excluded. If both the eyes of a patient were diagnosed with NTG, only the right eye was included in the study.

\subsection{OCTA Measurements}

OCTA measurements (Optovue Inc., Fremont, CA, USA) were performed at baseline and 6 months after topical carteolol treatment in the study subjects (sample size $\mathrm{n}=80$ ). After each scan, motion artifacts were corrected using an orthogonal registration algorithm. The microvasculature of the optic disc and peripapillary superficial retina was analyzed using the Angio Disc protocol installed within Optovue OCTA. Peripapillary superficial retinal VD was defined as a 750-micrometer-wide elliptical annulus extending from the optic disc boundary. Peripapillary VD in each Garway-Heath sector was calculated automatically (Figure 1D). 

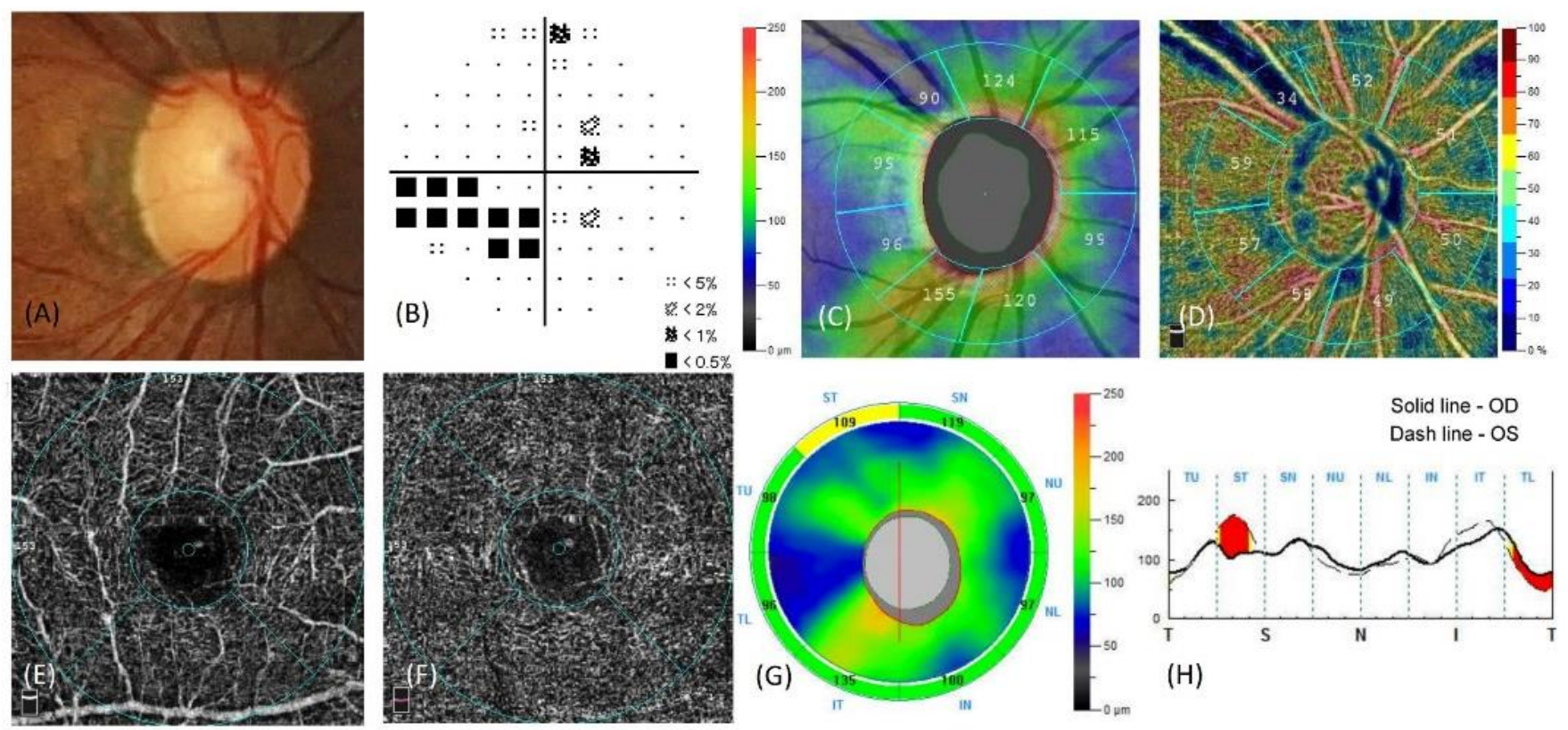

(H)

Figure 1. Representative case of a normal-tension glaucoma eye treated with carteolol (right eye). (A) The color photograph shows superior-temporal rim thinning of the optic disc. (B) The 30-2 Swedish interactive threshold algorithm standard pattern deviation image obtained using the Humphrey Visual Field Analyzer shows inferior-nasal step visual field defects (visual field mean deviation: $-6.54 \mathrm{~dB}$ ). (C) The circumpapillary retinal nerve fiber layer thickness map shows thinning at the superior-temporal regions. (D) The OCTA vessel density image shows apparent microvascular reduction at superiortemporal regions around the optic disc. (E) The superficial layer of the retina was measured in the 3.0 $\times 3.0-\mathrm{mm}$ region centered on the fovea. The boundary of the superficial retinal layer extends from the internal limiting membrane to the inner plexiform layer. (F) The deep retinal layer measured in the $3.0 \times 3.0-\mathrm{mm}$ region centered on the fovea. The boundary of the deep retinal layer extends from the inner to outer plexiform layers. (G) The retinal nerve fiber layer (RNFL) thickness map shows decreased RNFL thickness at the superior-temporal sector. (H) Significantly decreased RNFL thickness compared with the fellow eye at the superior-temporal sector.

At the fovea, the superficial and deep VDs of the retina were measured in the $3.0 \times 3.0-\mathrm{mm}$ region centered on the fovea. The boundary of the superficial retinal layer extended from the internal limiting membrane to the inner plexiform layer, and the boundary of the deep retinal layer extended from the inner to the outer plexiform layers.

Previous studies demonstrated that SSI values of the scans had a significant effect on the repeatability of OCTA, with the VD increasing in scans with higher SSI values $[10,11]$. To adjust the VD according to the SSI, the peripapillary VD/SSI was used for statistical analysis.

\subsection{Statistical Analysis}

Statistical analyses were performed using IBM SPSS Statistics for Windows/Macintosh, Version 20.0 (IBM Corp., Armonk, NY, USA). Variables in the current study are presented as means and standard deviations. The factors that may affect the percentage change in VD/SSI were evaluated using generalized estimating equations. Variables with a $p$ value $<0.2$ in the univariate analysis were included in the multivariate analysis. Statistical significance was set at $p<0.05$.

\section{Results}

A total of 115 patients with newly diagnosed NTG treated using topical carteolol were recruited for the initial evaluation. Among them, 35 were excluded based on the exclusion criteria. Finally, 80 patients (46 men and 34 women) were included in the analysis. The OCTA images of a representative case were shown in Figure 1. Table 1 summarizes the demographic and clinical characteristics of the included patients. Their mean age 
was $59.7 \pm 11.4$ years old, the mean CCT was $536.1 \pm 39.5 \mu \mathrm{m}$, the mean axial length was $25 \pm 1.6 \mathrm{~mm}$, the mean $\log$ MAR visual acuity was $0.27 \pm 0.27$, and the mean baseline IOP was $15.2 \pm 2.5 \mathrm{mmHg}$. The mean VF mean deviation was $-4.8 \pm 4.5 \mathrm{~dB}$, and the mean VF pattern standard deviation was $5.6 \pm 3.8 \mathrm{~dB}$. A negative correlation between the disc/cup ratio and the peripapillary VD was disclosed in Figure 2. On the other hand, the correlation between the VF mean deviation and the peripapillary VD was not significant $(\mathrm{r}=-0.01$, $p=0.928)$.

Table 1. Baseline characteristics of normal-tension glaucoma patients in this study.

\begin{tabular}{cccc}
\hline $\mathbf{n}=\mathbf{8 0}$ & Mean & SD & SEM \\
\hline Age (yrs) & 59.7 & 11.4 & 1.28 \\
CCT (um) & 536.1 & 39.5 & 4.40 \\
Axial length (mm) & 25 & 1.6 & 0.18 \\
VA(logMAR) & 0.27 & 0.27 & 0.03 \\
IOP(mmHg) & 15.2 & 2.5 & 0.27 \\
MD (dB) & -4.8 & 4.5 & 0.50 \\
PSD (dB) & 5.6 & 3.8 & 0.43
\end{tabular}

$\overline{\mathrm{SD}}=$ standard deviation, $\mathrm{CCT}=$ central corneal thickness, $\mathrm{VA}=$ visual acuity, IOP $=$ intraocular pressure $\mathrm{MD}=$ mean deviation, $\mathrm{PSD}=$ pattern standard deviation .

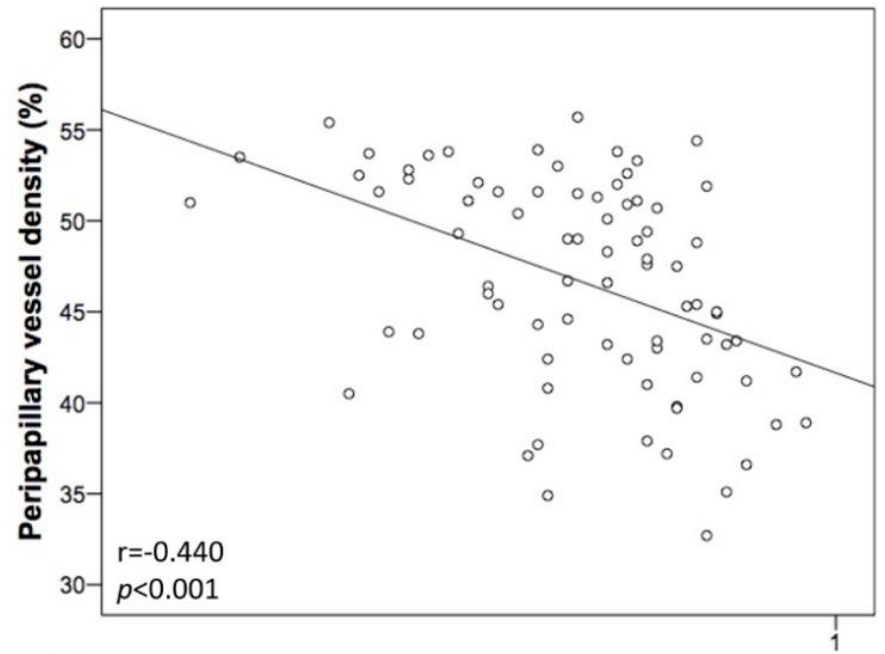

(A)

\section{Cup/Disc vertical ratio}

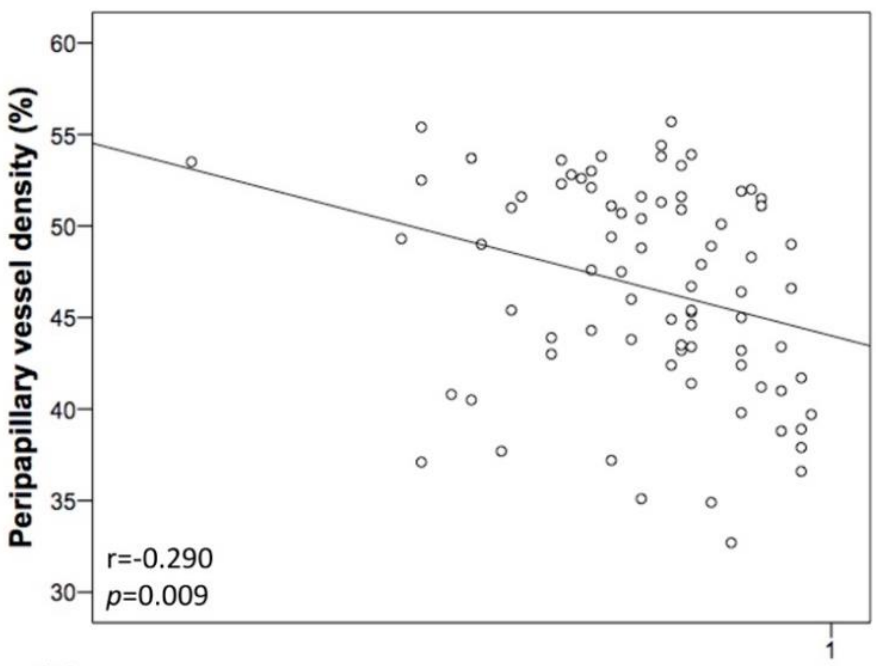

(B)
Cup/Disc horizontal ratio

Figure 2. Scatterplots between cup/disc vertical ratio and peripapillary vessel density (A); between cup/disc horizontal ratio and peripapillary vessel density $(\mathbf{B})$ in patients with normal-tension glaucoma treated with carteolol. (Sample size $\mathrm{n}=80$, r: Pearson correlation coefficient, $p: p$ value of Pearson correlation).

After the 6-month topical carteolol treatment, the peripapillary VD in 40 patients increased, whereas that in the other 40 patients decreased. Figure 3 shows the OCTA images acquired from two patients included in the current study. One patient showed an increased VD after the carteolol treatment (Figure 3A,B), and the other showed a decreased VD after the carteolol treatment (Figure 3C,D). Univariate analysis revealed that age and hypertension were significant factors that affected the percentage change in VD/SSI, and this result was confirmed in the multivariate analysis (Table 2). Older patients and those with hypertension were more likely to have a VD decrease after the 6-month topical carteolol treatment (Figures 4 and 5).

In addition, the baseline VD/SSI significantly affected the percentage change in the VD/SSI after the 6-month topical carteolol treatment (Table 3). In the univariate analysis, the VD/SSI in the peripapillary area, including the superior-nasal, superior-temporal, inferior-nasal, inferior-temporal, temporal, and nasal sectors, and in the superficial and 
deep macular areas all significantly affected the percentage change in the peripapillary VD/SSI. In the multivariate analysis, we adjusted for age, hypertension, pattern standard deviation, CCT, and the percentage change in IOP, and we found that the VD/SSI in all the peripapillary sectors and the superficial and deep macular areas still significantly affected the percentage change in the peripapillary VD/SSI. The patients with a higher initial VD/SSI in the peripapillary and macular areas were more likely to have a decreased peripapillary VD/SSI after the 6-month topical carteolol treatment (Figure 6).
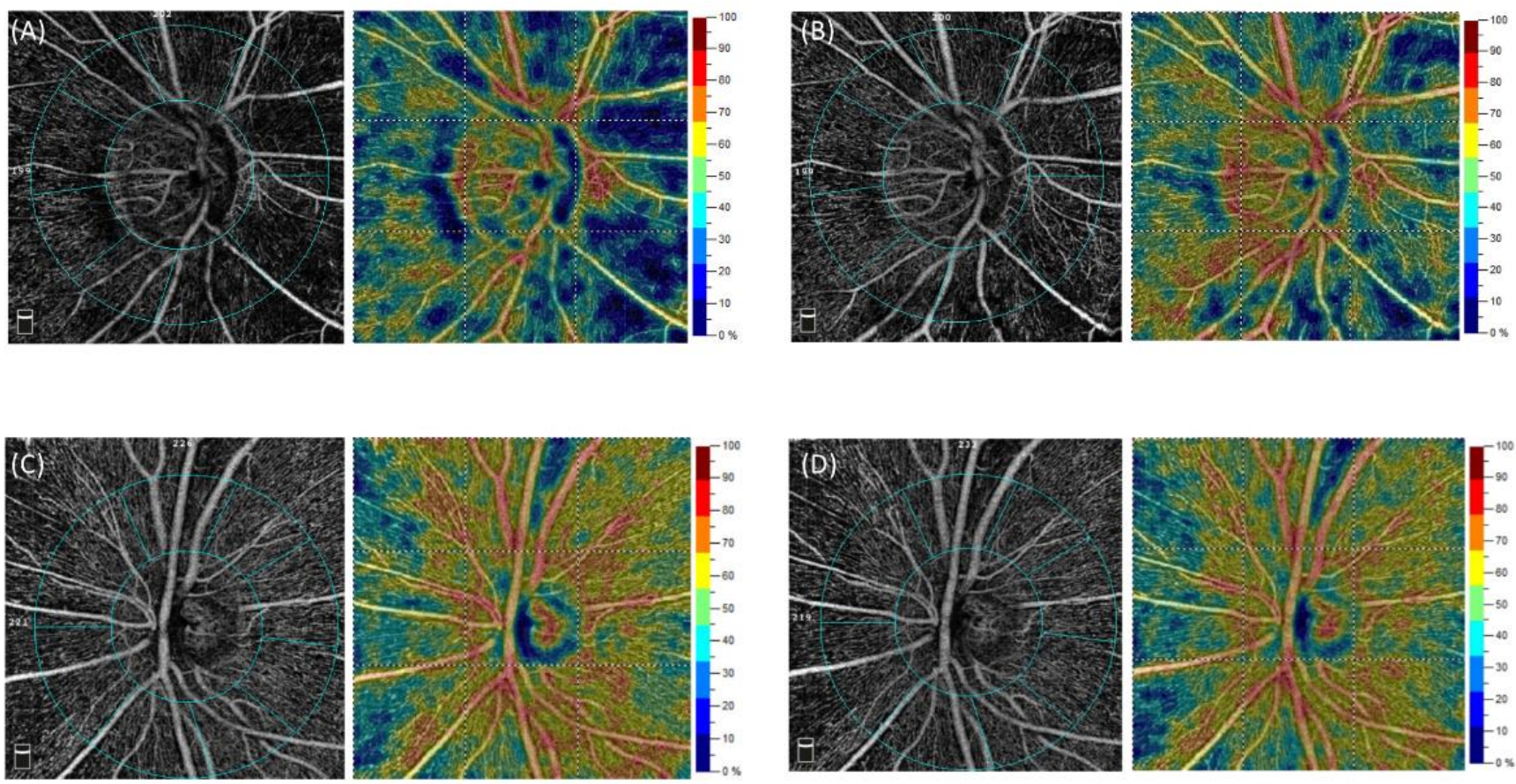

Figure 3. Representative cases of vessel density (VD) changes in patients with normal-tension glaucoma treated using carteolol. (A) Case 1, before carteolol treatment (peripapillary VD =37.9\%). (B) Case 1, VD increases after the 6-month topical carteolol treatment (peripapillary VD $=45.6 \%$ ). (C) Case 2, before carteolol treatment (peripapillary VD $=55.4 \%$ ).

(D) Case 2, VD decreases after the 6-month topical carteolol treatment (peripapillary VD $=52.4 \%$ ).

Table 2. Significant independent variables related to the change percentage of peripapillary vessel density/signal strength index after 6-month carteolol treatment in normal-tension glaucoma patients.

\begin{tabular}{|c|c|c|c|c|c|c|}
\hline & \multicolumn{3}{|c|}{ Univariate } & \multicolumn{3}{|c|}{ Multivariate } \\
\hline & B & $(95 \% \mathrm{CI})$ & $p$ Value & B & $(95 \% \mathrm{CI})$ & $p$ Value \\
\hline Age & -0.718 & $(-1.254,-0.182)$ & 0.009 & -0.644 & $(-1.253,-0.036)$ & 0.038 \\
\hline CCT & -0.159 & $(-0.354,0.037)$ & 0.111 & -0.109 & $(-0.293,0.075)$ & 0.247 \\
\hline Axial length & 0.345 & $(-4.221,4.911)$ & 0.882 & - & & \\
\hline VA & -7.999 & $(-33.166,17.169)$ & 0.533 & - & & \\
\hline IOP & -0.827 & $(-3.590,1.935)$ & 0.557 & - & & \\
\hline IOP change percentage & 0.211 & $(-0.103,0.526)$ & 0.188 & 0.111 & $(-0.216,0.438)$ & 0.507 \\
\hline MD & 0.358 & $(-0.886,1.602)$ & 0.573 & - & & \\
\hline PSD & 1.122 & $(-0.492,2.737)$ & 0.173 & 0.77 & $(-0.661,2.201)$ & 0.292 \\
\hline Hypertension & -14.415 & $(-27.816,-1.014)$ & 0.035 & -12.483 & $(-24.569,-0.397)$ & 0.043 \\
\hline Gender & 0.336 & $(-12.923,13.596)$ & 0.96 & - & & \\
\hline
\end{tabular}

$\mathrm{B}=$ coefficient of the associated factors in the generalized estimating equations, $\mathrm{CI}=$ confidence interval, $\mathrm{CCT}=$ central corneal thickness,

$\mathrm{VA}=$ visual acuity, $\mathrm{IOP}=$ intraocular pressure, $\mathrm{MD}=$ mean deviation, $\mathrm{PSD}=$ pattern standard deviation. 


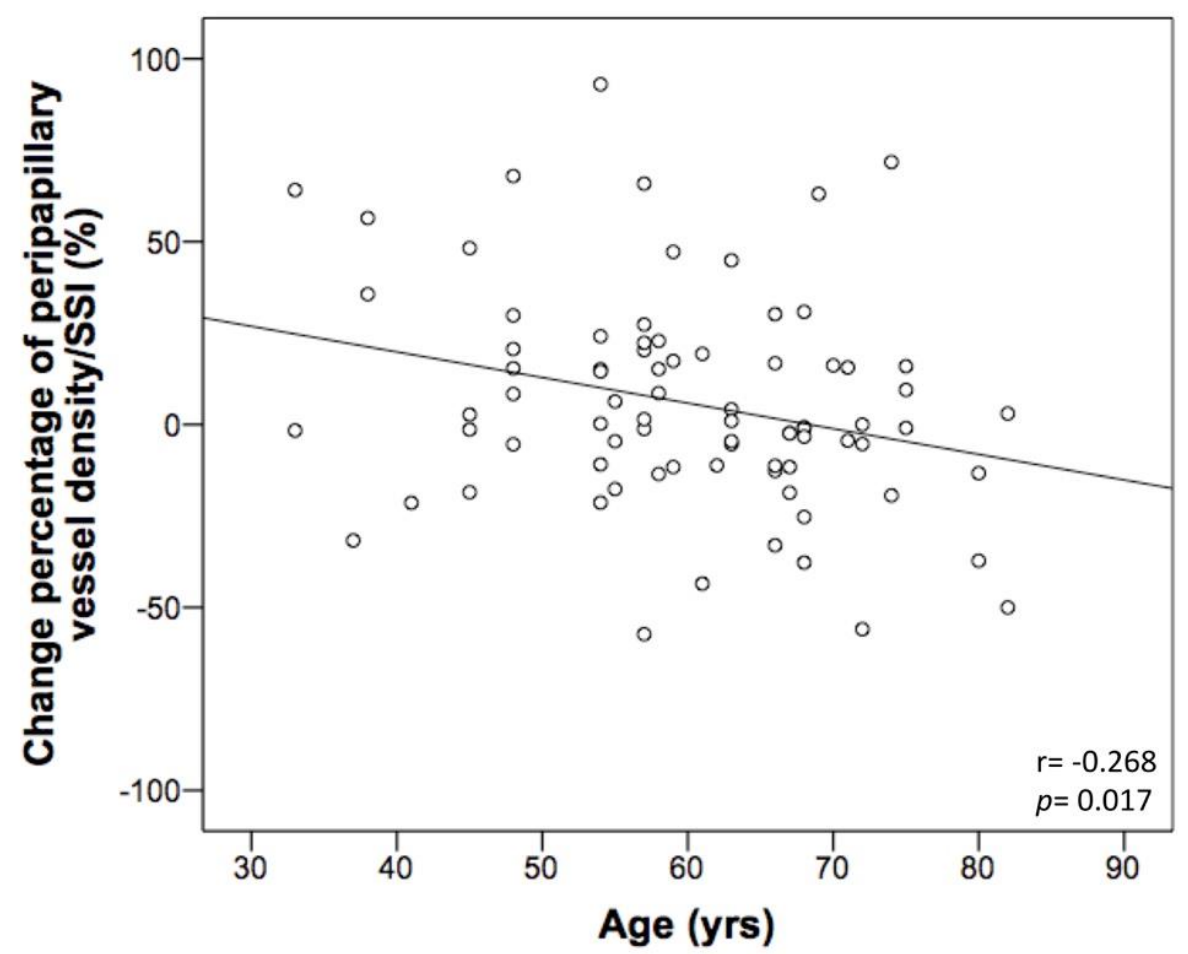

Figure 4. Scatterplot between age and percentage change in peripapillary vessel density/signal strength index (SSI) after the 6-month topical carteolol treatment in patients with normal-tension glaucoma. (Sample size $\mathrm{n}=80$, r: Pearson correlation coefficient, $p$ : $p$ value of Pearson correlation).

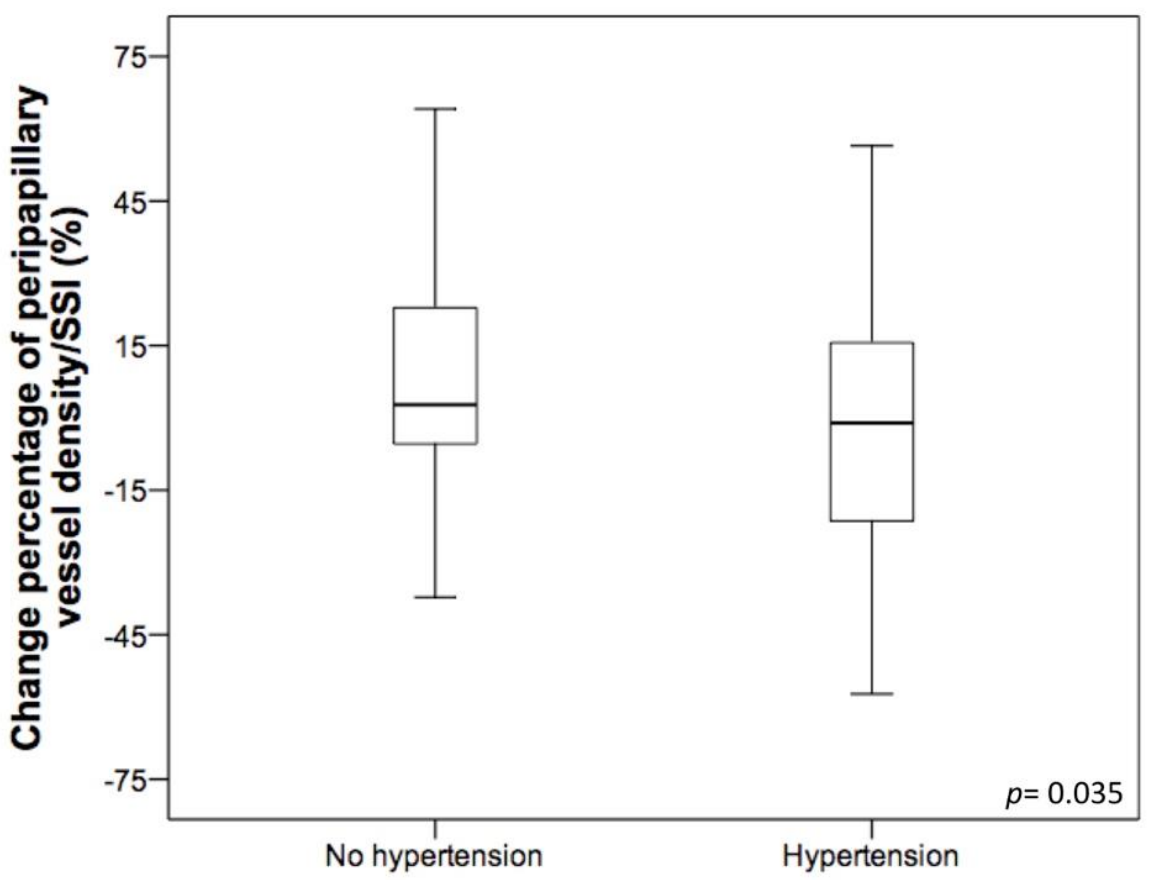

Figure 5. Percentage change in peripapillary vessel density/signal strength index (SSI) after the 6-month topical carteolol treatment in patients with normal-tension glaucoma with and without hypertension. (Sample size: no hypertension $\mathrm{n}=53$, hypertension $\mathrm{n}=27, p$ : $p$ value of univariate analysis). 
Table 3. Significant initial vessel density/SSI related to the change percentage of peripapillary vessel density/signal strength index after 6-month carteolol treatment in normal-tension glaucoma patients.

\begin{tabular}{|c|c|c|c|c|c|c|}
\hline & \multicolumn{3}{|c|}{ Univariate } & \multicolumn{3}{|c|}{ Multivariate } \\
\hline & B & $(95 \% \mathrm{CI})$ & $p$ Value & B & $(95 \% \mathrm{CI})$ & $p$ Value \\
\hline Peripapillary VD/SSI & -69.06 & $(-94.11,-44.00)$ & $<0.001$ & -64.94 & $(-89.72,-40.17)$ & $<0.001$ \\
\hline SN peripapillary VD/SSI & -54.66 & $(-78.38,-35.95)$ & $<0.001$ & -52.45 & $(-68.97,-35.93)$ & $<0.001$ \\
\hline ST peripapillary VD/SSI & -53.81 & $(-72.19,-35.43)$ & $<0.001$ & -51.95 & $(-68.97,-33.94)$ & $<0.001$ \\
\hline $\mathrm{T}$ peripapillary VD/SSI & -58.84 & $(-85.21,-32.47)$ & $<0.001$ & -53.4 & $(-80.96,-25.85)$ & $<0.001$ \\
\hline IT peripapillary VD/SSI & -43.34 & $(-54.22,-32.67)$ & $<0.001$ & -42.62 & $(-55.32,-29.92)$ & $<0.001$ \\
\hline IN peripapillary VD/SSI & -66.07 & $(-90.95,-41.19)$ & $<0.001$ & -62.91 & $(-86.85,-38.94)$ & $<0.001$ \\
\hline $\mathrm{N}$ peripapillary VD/SSI & -56.17 & $(-75.57,-36.77)$ & $<0.001$ & -52.52 & $(-73.03,-32.01)$ & $<0.001$ \\
\hline Superficial macula VD/SSI & -69.34 & $(-97.03,-41.66)$ & $<0.001$ & -66.88 & $(-95.66,-38.10)$ & $<0.001$ \\
\hline Deep macula VD/SSI & -48.93 & $(-80.31,-17.55)$ & 0.002 & -45.14 & $(-76.54,-13.75)$ & 0.005 \\
\hline
\end{tabular}

$\mathrm{B}=$ coefficient of the associated factors in the generalized estimating equations, $\mathrm{CI}=$ confidence interval, $\mathrm{VD}=$ vessel density, $\mathrm{SSI}=$ signal strength index, $\mathrm{SN}=$ superior nasal, $\mathrm{ST}=$ superior temporal, $\mathrm{T}=$ temporal, $\mathrm{IT}=$ inferior temporal, $\mathrm{IN}=$ inferior nasal, $\mathrm{N}=$ nasal.
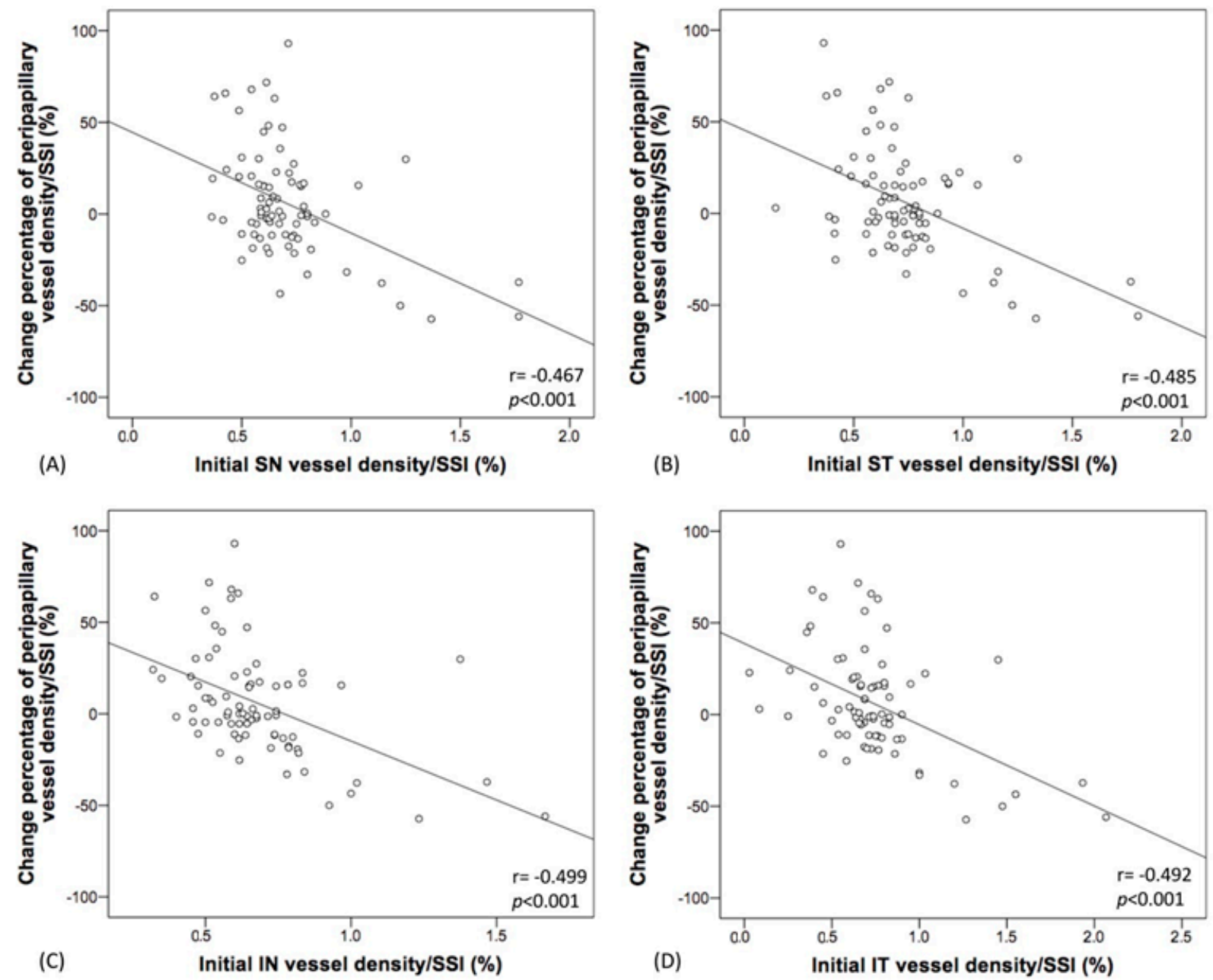

Figure 6. Scatterplots between initial superior nasal (SN) peripapillary vessel density (VD)/signal strength index (SSI) and percentage change in peripapillary VD/SSI (A); between initial superior temporal (ST) peripapillary VD/SSI and percentage change in peripapillary VD/SSI (B); between initial inferior nasal (IN) peripapillary VD/SSI and percentage change in peripapillary VD/SSI (C); and between initial inferior temporal (IT) peripapillary VD/SSI and percentage change in peripapillary VD/SSI (D) after the 6-month topical carteolol treatment in patients with normal-tension glaucoma. (Sample size $\mathrm{n}=80$, $\mathrm{r}$ : Pearson correlation coefficient, $p$ : $p$ value of Pearson correlation). 


\section{Discussion}

This study indicated that age, hypertension, and the initial VD/SSI were associated with the percentage changes in peripapillary VD/SSI after 6 months of topical carteolol treatment in eyes with NTG. To our knowledge, this is the first study to evaluate the changes in VD after topical carteolol treatment in patients with glaucoma.

Carteolol is a nonselective $\beta$-blocker with intrinsic sympathomimetic activity (ISA). $\beta$ blockers directly block vascular $\beta 2$-adrenoceptors, resulting in vasoconstriction. $\beta$-blockers with ISA can stimulate $\beta$-adrenoceptors (via an agonistic effect). When compared to a full $\beta$-adrenoceptor agonist such as timolol, a $\beta$-blocker with ISA will only induce a submaximal response when bound to receptors at maximal occupancy [12]. The presence of ISA in a $\beta$-blocker modifies its effects on peripheral circulation. Peripheral blood flow is decreased to a lesser extent by a $\beta$-blocker with ISA than by one without ISA [13]. In addition, carteolol can act as a serotonin 5-HT1A and 5-HT1B receptor antagonist [14]. 5-HT1B receptors mediate the contraction of vascular smooth muscles. Thus, carteolol may lead to vessel dilation by blocking 5-HT1B receptors [15].

To date, the effect of carteolol on retinal perfusion in patients with glaucoma has remained controversial. In our study, the peripapillary VD increased in 40 patients but decreased in the other 40 patients after the 6-month topical carteolol treatment. In 2001, Montanari et al. evaluated 20 patients with bilateral POAG by using color Doppler imaging. After treatment with $2 \%$ carteolol, the resistance index of the short posterior ciliary arteries was significantly reduced. In addition, $2 \%$ carteolol caused significant changes in the VF indices, with an increase in the mean VF sensitivity and a decrease in mean deviation. Their report suggested that the ISA of carteolol may reduce peripheral vascular resistance, thereby improving perfusion to the ONH [16]. In 2012, Kawai et al. used laser Doppler velocimetry to evaluate retinal arterial blood flow (RBF) in patients with POAG treated using carteolol. They found that carteolol preserved RBF in 16 patients, but it decreased RBF with low blood pressure in four patients, suggesting that topical carteolol treatment decreased RBF in some patients with decreased ocular perfusion [17].

After analyzing the factors that affected the changes in VD, we found that the initial peripapillary and macular VD/SSIs correlated with the percentage change in peripapillary VD/SSI. The patients with NTG and a higher initial VD/SSI were more likely to have decreased peripapillary VD/SSI after the 6-month topical carteolol treatment. Previous studies have demonstrated that $\beta$-blockers directly affect vascular $\beta 2$-adrenoceptors, resulting in vasoconstriction [18], and such receptors have been detected in both bovine [19] and human retinal vessels [20]. Thus, it is reasonable that patients with more abundant retinal vascularity have more vascular $\beta 2$-adrenoceptors on the retina, and they will be affected more significantly by $\beta$-blocker instillation than would those with less abundant retinal vascularity. Therefore, the retinal blood flow will be affected more in those with high retinal vessel density.

In addition, we found that older patients with NTG and hypertension were more likely to have a decrease in VD after the 6-month topical carteolol treatment. We did not have sufficient data to explain the mechanisms underlying this observation. However, the findings implied that older patients and those with hypertension have higher vascular resistance [21], and the carteolol ISA effect and its binding capacity to 5-HT1B receptors might be altered in these individuals, thus resulting in more pronounced retinal vasoconstriction after carteolol treatment.

Despite its strengths, our study has some limitations. First, the sample size was relatively small, and the study included patients of a single ethnicity. Second, we did not have sufficient data to explain the mechanisms underlying the observation that older age and hypertension could affect VD changes in patients with NTG treated using carteolol. Thus, future large-scale studies or animal studies are warranted to confirm the findings of this study. 


\section{Conclusions}

Carteolol treatment could lead to an increase in VD in $50 \%$ of patients with NTG and a decrease in VD in the other 50\%. The baseline VD, age, and hypertension could affect VD changes. Patients with NTG with higher baseline peripapillary or macular VD, older age, and hypertension are more likely to have decreased VD after carteolol treatment. Therefore, clinicians should be aware of this possibility when treating patients with NTG by using topical carteolol.

Author Contributions: Conceptualization, Y.-H.L. and L.-C.C.; data curation, L.-H.C.; formal analysis, Y.-H.L.; investigation, S.-M.H.; methodology, S.-M.H. and L.-H.C.; resources, L.-C.C.; software, Y.-H.L. and S.-M.H.; supervision, L.-C.C.; validation, Y.-H.L., S.-M.H., L.-H.C. and L.-C.C.; visualization, L.-C.C.; writing-original draft, Y.-H.L. and S.-M.H.; writing-review and editing, Y.-H.L., S.-M.H., L.-H.C. and L.-C.C. All authors have read and agreed to the published version of the manuscript.

Funding: This research received no external funding.

Institutional Review Board Statement: The study was conducted according to the guidelines of the Declaration of Helsinki, and approved by the Institutional Review Board of Chang Gung Memorial Hospital (IRB approval number: 202000935B0 and date of approval: 1 July 2020).

Informed Consent Statement: Informed consent was obtained from all the subjects involved in the study.

Data Availability Statement: The clinical data used to support the findings of this study are included within the article.

Acknowledgments: We extend our deepest gratitude to Hsing-Fen Lin for offering invaluable advice and informative suggestions regarding statistical analysis.

Conflicts of Interest: The authors declare that there is no conflict of interest.

\section{References}

1. Augusto, A.B.; Luca, B.; Alessandro, B.; Keith, B. European Glaucoma Society Terminology and Guidelines for Glaucoma, 4th Edition. Br. J. Ophthalmol. 2017, 101, 130-195. [CrossRef]

2. Kaiser, H.J.; Schoetzau, A.; Stumpfig, D.; Flammer, J. Blood-flow velocities of the extraocular vessels in patients with high-tension and normal-tension primary open-angle glaucoma. Am. J. Ophthalmol. 1997, 123, 320-327. [CrossRef]

3. Flammer, J.; Konieczka, K.; Flammer, A.J. The primary vascular dysregulation syndrome: Implications for eye diseases. EPMA J. 2013, 4, 14. [CrossRef] [PubMed]

4. Tsai, J.C. Influencing ocular blood flow in glaucoma patients: The cardiovascular system and healthy lifestyle choices. Can. J. Ophthalmol. 2008, 43, 347-350. [CrossRef] [PubMed]

5. Triolo, G.; Rabiolo, A. Optical coherence tomography and optical coherence tomography angiography in glaucoma: Diagnosis, progression, and correlation with functional tests. Ther. Adv. Ophthalmol. 2020, 12, 2515841419899822. [CrossRef] [PubMed]

6. Bekkers, A.; Borren, N.; Ederveen, V.; Fokkinga, E.; Andrade De Jesus, D.; Sanchez Brea, L.; Klein, S.; van Walsum, T.; BarbosaBreda, J.; Stalmans, I. Microvascular damage assessed by optical coherence tomography angiography for glaucoma diagnosis: A systematic review of the most discriminative regions. Acta Ophthalmol. 2020, 98, 537-558. [CrossRef]

7. Van Melkebeke, L.; Barbosa-Breda, J.; Huygens, M.; Stalmans, I. Optical Coherence Tomography Angiography in Glaucoma: A Review. Ophthalmic Res. 2018, 60, 139-151. [CrossRef]

8. Renard, P.; Kovalski, J.L.; Cochereau, I.; Jaulerry, S.; Williamson, W.; Elena, P.P.; Lablache Combier, M.; Allaire, C.; Siou-Mermet, R. Comparison of carteolol plasmatic levels after repeated instillations of long-acting and regular formulations of carteolol $2 \%$ in glaucoma patients. Graefes Arch. Clin. Exp. Ophthalmol. 2005, 243, 1221-1227. [CrossRef]

9. Chihara, E.; Dimitrova, G.; Chihara, T. Increase in the OCT angiographic peripapillary vessel density by ROCK inhibitor ripasudil instillation: A comparison with brimonidine. Graefes Arch. Clin. Exp. Ophthalmol. 2018, 256, 1257-1264. [CrossRef]

10. Venugopal, J.P.; Rao, H.L.; Weinreb, R.N.; Dasari, S.; Riyazuddin, M.; Pradhan, Z.S.; Puttaiah, N.K.; Devi, S.; Mansouri, K.; Webers, C.A.B. Repeatability and comparability of peripapillary vessel density measurements of high-density and non-high-density optical coherence tomography angiography scans in normal and glaucoma eyes. Br. J. Ophthalmol. 2019, 103, 949-954. [CrossRef]

11. Venugopal, J.P.; Rao, H.L.; Weinreb, R.N.; Pradhan, Z.S.; Dasari, S.; Riyazuddin, M.; Puttiah, N.K.; Rao, D.A.S.; Devi, S.; Mansouri, K.; et al. Repeatability of vessel density measurements of optical coherence tomography angiography in normal and glaucoma eyes. Br. J. Ophthalmol. 2018, 102, 352-357. [CrossRef]

12. Jaillon, P. Relevance of intrinsic sympathomimetic activity for beta blockers. Am. J. Cardiol. 1990, 66, 21C-23C. [CrossRef] 
13. Ireland, M.A.; Littler, W.A. The effects of oral accbutolol and propranolol on forearm blood flow in hypertensive patients. Br. J. Clin. Pharmacol. 1981, 12, 363-368. [CrossRef]

14. Langlois, M.; Bremont, B.; Rousselle, D.; Gaudy, F. Structural analysis by the comparative molecular field analysis method of the affinity of beta-adrenoreceptor blocking agents for 5-HT1A and 5-HT1B receptors. Eur. J. Pharmacol. 1993, 244, 77-87. [CrossRef]

15. De Vries, P.; Sanchez-Lopez, A.; Centurion, D.; Heiligers, J.P.; Saxena, P.R.; Villalon, C.M. The canine external carotid vasoconstrictor 5-HT1 receptor: Blockade by 5-HT1B (SB224289), but not by 5-HT1D (BRL15572) receptor antagonists. Eur. J. Pharmacol. 1998, 362, 69-72. [CrossRef]

16. Montanari, P.; Marangoni, P.; Oldani, A.; Ratiglia, R.; Raiteri, M.; Berardinelli, L. Color Doppler imaging study in patients with primary open-angle glaucoma treated with timolol 0.5\% and carteolol 2. Eur. J. Ophthalmol. 2001, 11, 240-244. [CrossRef] [PubMed]

17. Kawai, M.; Nagaoka, T.; Takahashi, A.; Sato, E.; Yoshida, A. Effects of topical carteolol on retinal arterial blood flow in primary open-angle glaucoma patients. Jpn. J. Ophthalmol. 2012, 56, 458-463. [CrossRef] [PubMed]

18. Cruickshank, J.M. The clinical importance of cardioselectivity and lipophilicity in beta blockers. Am. Heart J. 1980, 100, 160-178. [CrossRef]

19. Ferrari-Dileo, G. Beta 1 and beta 2 adrenergic binding sites in bovine retina and retinal blood vessels. Investig. Ophthalmol. Vis. Sci. 1988, 29, 695-699.

20. Denis, P.; Elena, P.P. Retinal vascular beta-adrenergic receptors in man. Ophtalmologie 1989, 3, 62-64.

21. Feihl, F.; Liaudet, L.; Levy, B.I.; Waeber, B. Hypertension and microvascular remodelling. Cardiovasc. Res. 2008, 78, 274-285. [CrossRef] [PubMed] 